\title{
Indução da ovulação em cabras, fora da estação reprodutiva, com LH e GnRH e com estro induzido por progestágenos
}

[Induction of the out-of-season ovulation (with LH and GnRH) and estrus (with progesterone) in goats]

\author{
P.A.G. Leite $^{1}$, G.R. Carvalho ${ }^{2}$, M.T. Rodrigues ${ }^{2}$, J.R.M. Ruas ${ }^{3}$, E.A.M. Amorim ${ }^{4}$, V.V. Maffili ${ }^{5}$ \\ ${ }^{1}$ Médico veterinário autônomo \\ ${ }^{2}$ Departamento de Zootecnia - UFV \\ Avenida P. H. Rolfs s/n \\ 36570-000 - Viçosa, MG \\ ${ }^{3}$ EPAMIG - Viçosa, MG \\ ${ }^{4}$ Aluno de pós-graduação - UFV - Viçosa, MG \\ ${ }^{5}$ FIOCRUZ - Rio de Janeiro, RJ
}

\begin{abstract}
RESUMO
Quarenta e cinco cabras foram sincronizadas com o uso de esponjas intravaginais impregnadas com 60mg de acetato de medroxiprogesterona (MAP) por nove dias, associadas com 200UI de gonadotrofina coriônica equina (eCG) e 37,5 $\mathrm{g}$ de cloprostenol no sétimo dia. Os animais foram separados em três grupos e receberam, 24 horas após a retirada das esponjas, solução salina ou hormônios, no seguinte esquema: $\mathrm{T}_{1}$ (controle) - $1 \mathrm{ml}$ de solução salina; $\mathrm{T}_{2}-5 \mathrm{mg}$ de hormônio luteinizante $(\mathrm{LH})$ e $\mathrm{T}_{3}-12,5 \mu \mathrm{g}$ do hormônio liberador de gonadotrofina $(\mathrm{GnRH})$. Houve diferença $(\mathrm{P}<0,05)$ na porcentagem de animais em estro, $100,0 \%, 73,3 \%$ e $66,6 \%$, para $\mathrm{T}_{1}, \mathrm{~T}_{2}$ e $\mathrm{T}_{3}$, respectivamente. Na mesma ordem, não houve diferença $(\mathrm{P}>0,05)$ quanto ao intervalo da retirada da esponja ao aparecimento do estro, que foi, em média, 34,8, 29,3 e 31,5 horas, respectivamente. O intervalo médio da retirada da esponja à ovulação foi de 46,6, 52,1 e 41,6 horas, respectivamente, com diferença significativa entre $T_{2}$ e $T_{3}(P<0,01)$. As ovulações ocorreram, em média, 21,3, 26,8 e 22,3 horas após as aplicações ( $\mathrm{P}>0,05)$. A aplicação de LH e do GnRH para induzir e sincronizar a ovulação em cabras fora da estação reprodutiva não apresentou resultados satisfatórios.
\end{abstract}

Palavras-chave: cabra, sincronização, cio, ovulação, LH, GnRH, MAP

\begin{abstract}
In order to achieve estrous synchronization, intravaginal sponges impregnated with 60mg of medroxyprogesterone acetate (MPA) were implanted in 45 goats for nine days. On day 7, 200IU of equine chorionic gonadotropin (eCG) and $37.5 \mu \mathrm{g}$ of cloprostenol were administered to each doe. The animals were randomly allocated in three treatments with 15 animals each. Twenty four hours after intravaginal sponge removal, does were administered $1 \mathrm{ml}$ saline solution (control $\left.T_{1}\right)$, $5 \mathrm{mg} \mathrm{LH}\left(\mathrm{T}_{2}\right)$ and

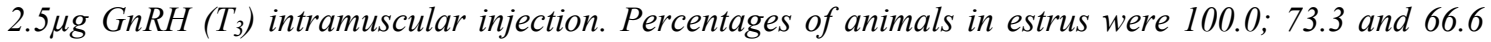
$(P<0.05)$ and average intervals from sponge removal to start of estrus were 34.8, 29.3 and 31.5 hours for $T_{1}, T_{2}$ and $T_{3}$, respectively, being those differences not statistically significant. The average intervals from sponge removal to ovulation were 46.6, 52.1 and 41.6 hours for $T_{1}, T_{2}$ and $T_{3}$, respectively, with the difference between $T_{2}$ and $T_{3}$ being statistically significant $(P<0.01)$. Ovulation time averages were 21.3, 26.8 and 22.3 hours after injections for $T_{1}, T_{2}$ and $T_{3}$, respectively.
\end{abstract}

Keywords: goat, synchronization, ovulation, $L H$, GnRH, MPA

Recebido em 18 de maio de 2004

Aceito em 21 de setembro de 2005

*Autor para correspondência (corresponding author)

E-mail: giovanni@ufv.br 


\section{INTRODUÇ̃̃O}

Os caprinos, como outros pequenos ruminantes, particularmente em regiões de clima temperado, apresentam marcada sazonalidade sexual, que se manifesta tanto na fêmea como no macho (Shelton, 1978). Nessas condições, os caprinos são classificados como poliéstricos estacionais de dias curtos. Nessas regiões apresentam comportamento reprodutivo influenciado positivamente pela diminuição das horas de luz do dia. Em regiões onde as mudanças do fotoperíodo são mínimas, o comportamento reprodutivo é mais influenciado pela alimentação e pela temperatura ambiente (Chemineau et al., 1991).

A ocorrência de anestro estacional limita o desempenho produtivo dos caprinos, visto que apenas se consegue um parto/fêmea/ano, assim como a inexistência de planejamento na exploração leiteira, em regiões de alta latitude, tem concentrado sua produção em determinados meses do ano.

A reprodução programada, por maximizar tanto a produção de leite quanto a eficiência reprodutiva do rebanho, vem sendo implementada por algumas biotécnicas, como a indução e sincronização do estro, a inseminação artificial e os métodos de diagnóstico precoce de gestação (Baril, 1995). Tratamentos hormonais para sincronização e/ou indução do estro têm sido usados para auxiliar na inseminação artificial (IA) e para reduzir os efeitos da sazonalidade sobre o desempenho reprodutivo das fêmeas caprinas. Os métodos mais utilizados combinam o uso de esponjas intravaginais impregnadas com progestágenos com injeção de gonadotrofina coriônica eqüina (eCG) e um análogo da prostaglandina $\mathrm{PGF}_{2 \alpha}$ (cloprostenol) aplicado até 48 horas antes da remoção das esponjas (Freitas et al., 1996).

O controle do estro e da ovulação de animais continua sendo a base e o pré-requisito para o sucesso do controle reprodutivo (Motlomelo et al., 2002). A sincronização, a identificação do momento da ovulação e a determinação da correlação entre a duração do estro e momento da ovulação são de grande importância para o estabelecimento de protocolos efetivos de inseminação artificial em tempo fixo, que resultem em máxima fertilidade ao parto.

O objetivo deste estudo foi avaliar o efeito da administração de LH e GnRH sobre a indução e a sincronização da ovulação em cabras fora da estação reprodutiva.

\section{MATERIAL E MÉTODOS}

O experimento foi realizado em Viçosa, Zona da Mata Norte do estado de Minas Gerais, situada a $20^{\circ} 45^{\prime} 14^{\prime}$ ' latitude sul e $42^{\circ} 52^{\prime} 53^{\prime}$ ' longitude oeste, altitude média de $648,74 \mathrm{~m}$, clima Cwa, pela classificação de Köppen (inverno seco e verão úmido), com temperatura média anual de $20,9^{\circ} \mathrm{C}$ e precipitação pluvial anual de $1203 \mathrm{~mm}^{3}$. $\mathrm{O}$ período experimental compreendeu os meses de janeiro e fevereiro de 2003 (estação nãoreprodutiva dos caprinos, criados nessa região). Os dados de temperatura e umidade foram aferidos diariamente no local do experimento por meio de um aparelho termoigrógrafo ${ }^{1}$ e por termômetro de temperatura máxima e mínima.

Foram utilizadas 45 fêmeas pluríparas, lactantes (30 da raça Alpina e 15 da raça Saanen), com idades entre dois e seis anos, com peso corporal médio de $55,25 \pm 12,96 \mathrm{~kg}$, escore de condição corporal de 2,91 $\pm 1,05$ (escala de 1 a 5) e produção média de leite de $1,11 \pm 0,63 \mathrm{~kg}$. Os animais foram mantidos em regime intensivo durante o período experimental, alimentados com silagem de milho e ração concentrada misturada à mesma, fornecida duas vezes ao dia, além de sal mineral e água à vontade.

O ciclo estral foi induzido e sincronizado, inserindo-se esponjas intravaginais impregnadas com 60mg de acetato de medroxiprogesterona $(\mathrm{MAP})^{2}$, por um período de nove dias. No sétimo dia foram aplicadas por via intramuscular 200UI de gonadotrofina coriônica eqüina $(\mathrm{eCG})^{3}$ e $37,5 \mu \mathrm{g}$ de prostaglandina sintética (dcloprostenol) $)^{4}$.

\footnotetext{
${ }^{1}$ Termoigrógrafo Wallac, Wilh Lambrecht KG Götingen, Type 252

${ }^{2}$ Progespon ${ }^{\circledR}$, Syntex S.A., Indústria Bioquímica e

Farmacêutica, Buenos Aires, Argentina.

${ }^{3}$ Novormon ${ }^{\circledR}$ 5000, Syntex S.A., Indústria Bioquímica e

Farmacêutica, Buenos Aires, Argentina.

${ }^{4}$ Prolise ${ }^{\circledR}$, ARSA S. R. L., Buenos Aires, Argentina.
} 
Doze horas após a retirada das esponjas, iniciouse a observação das fêmeas, para registro daquelas em estro, a cada quatro horas, por um período de 20 minutos, com o auxílio de rufião. Vinte e quatro horas após a retirada das esponjas todas as cabras foram aleatoriamente distribuídas em três tratamentos $(\mathrm{T})$ com 15 animais cada: as do $\mathrm{T}_{1}$ receberam $1 \mathrm{ml}$ de solução salina; as do $\mathrm{T}_{2}$, $5 \mathrm{mg}$ de hormônio luteinizante $(\mathrm{LH})^{5}$; e as do $\mathrm{T}_{3}$, $12,5 \mu \mathrm{g}$ de análogo de hormônio liberador de gonadotrofina $(\mathrm{GnRH})^{6}$.

O desenvolvimento folicular ovariano foi acompanhado por ultra-sonografia via transretal, com transdutor linear de $5 \mathrm{MHz}^{7}$, próprio para humanos, acoplado a uma haste acrílica (diâmetro 25mm; comprimento de $50 \mathrm{~cm}$ ), inserido e manipulado no reto dos animais pela extensão dessa haste. Os exames foram realizados nos dias da colocação da esponja (dia 0), na aplicação da eCG e da $\mathrm{PGF}_{2 \alpha}$ (dia 7) e na retirada da esponja (dia 9). Doze horas após a retirada das esponjas, os exames foram feitos em intervalos de quatro horas e encerrados na detecção da ovulação e no término do estro. No exame foi avaliada a condição dos ovários, mensurados os folículos $(\geq 3 \mathrm{~mm}$ de diâmetro; Menchaca e Rubianes, 2002) e determinado o momento da ovulação, pelo colapso do maior folículo, usualmente com diâmetro $\geq 5 \mathrm{~mm}$ (Rubianes et al., 1996).

Utilizou-se sêmen de bodes com fertilidade comprovada. Determinadas as características seminais de volume, motilidade e vigor, o sêmen foi diluído em meio à base de leite desnatado. Todas as cabras foram inseminadas com sêmen fresco, contendo uma concentração de $100 \times 10^{6}$ de espermatozóides viáveis por dose (palheta de $0,5 \mathrm{ml}$ ). Foram realizadas duas inseminações artificiais pelo método transcervical, com o uso de aplicador universal e um espéculo vaginal (próprios para caprinos), em tempo fixo de $24 \mathrm{e}$ 36 horas após a retirada das esponjas. Ao $25^{\circ}$ dia após a IA, fez-se o diagnóstico de gestação, com auxílio da ultra-sonografia, confirmado ao $40^{\circ} \mathrm{e}$ $60^{\circ}$ dias.

Os dados do intervalo da retirada da esponja ao início do estro, da retirada da esponja à ovulação, a duração do estro, o intervalo do início do estro à ovulação e da aplicação da solução salina, ou do LH ou do GnRH, à ovulação foram submetidos à análise de variância. As médias foram comparadas pelo teste SNK, usando-se o SAEG (Sistema..., 1999). A taxa de gestação e a porcentagem de animais em estro foram comparadas usando-se o teste do qui-quadrado.

\section{RESULTADOS E DISCUSSÃO}

O estro foi eficientemente induzido e sincronizado em cabras fora da estação reprodutiva, obtendo-se $100,0 \%, 73,3 \%$ e $66,6 \%$ de estros no $\mathrm{T}_{1}, \mathrm{~T}_{2}$ e $\mathrm{T}_{3}$, respectivamente. Houve diferença $(\mathrm{P}<0,05)$ entre o primeiro e os dois últimos tratamentos. $\mathrm{O}$ intervalo da retirada da esponja ao início do estro e a duração do estro são apresentados na Tab. 1. Duas cabras do $T_{1}$ perderam a esponja intravaginal no período de indução e sincronização, sendo excluídas do experimento. As características avaliadas não diferiram $(\mathrm{P}>0,05)$ entre as duas raças.

Quatro cabras do $T_{2}$ e cinco do $T_{3}$ não apresentaram estro até 96 horas após a retirada das esponjas, apesar de todas terem ovulado. Esses animais foram excluídos da análise estatística para a porcentagem de animais em estro, intervalo da retirada da esponja ao início do estro, duração do estro e intervalo do início do estro à ovulação.

Vários estudos realizados com a utilização de esponjas impregnadas com 60mg de MAP, por um período variando de nove a 18 dias e associação de 200 a 500UI de eCG aplicada 48 horas antes ou no momento da retirada da esponja, associada ou não com a injeção de prostaglandina, mostraram valores superiores a 90,0\% de indução de estro (Greyling e van Niekerk, 1990; Baril et al., 1993; Menegatos et al., 1995; Zarkawi et al., 1999; Greyling e van der Nest, 2000; Fonseca, 2002). Os dados obtidos no presente estudo, em relação à indução de estro nos animais do $T_{2}$ e $T_{3}$, foram inferiores aos encontrados pelos autores citados.

\footnotetext{
${ }^{5}$ Lutropin ${ }^{\circledR}-\mathrm{V}$, Vetrepharm, Canadá Inc., Ontário, Canadá.

${ }^{6}$ Gestran Plus ${ }^{\circledR}$ ARSA S. R. L., Buenos Aires, Argentina.

${ }^{7}$ Aloka, modelo SSD - 500, Tókio, Japão.
} 
Tabela 1. Porcentagem de animais em estro, intervalo da retirada da esponja ao início do estro e duração do estro em cabras induzidas fora da estação reprodutiva com a utilização de MAP + eCG + cloprostenol associada à aplicação de solução salina $\left(\mathrm{T}_{1}\right)$, ou LH $\left(\mathrm{T}_{2}\right)$ ou GnRH $\left(\mathrm{T}_{3}\right)$, realizada 24 horas após a retirada da esponja (média \pm DP)

\begin{tabular}{|c|c|c|c|c|c|c|}
\hline \multirow[t]{2}{*}{ Tratamento } & \multicolumn{2}{|c|}{ Animais em estro } & \multicolumn{2}{|c|}{$\begin{array}{c}\text { Intervalo da retirada da } \\
\text { esponja ao estro }\end{array}$} & \multicolumn{2}{|c|}{ Duração do estro } \\
\hline & $\mathrm{n}$ & $(\%)$ & $\mathrm{n}$ & (horas) & $\mathrm{n}$ & (horas) \\
\hline$\overline{\mathrm{T}_{1}}$ & 13 & $100,00 \mathrm{~A}(13 / 13)$ & 13 & $34,8 \pm 10,4$ & 13 & $19,4 \pm 9,3$ \\
\hline $\mathrm{T}_{2}$ & 11 & $73,33 \mathrm{~B}(11 / 15)$ & 11 & $29,3 \pm 3,2$ & 11 & $19,7 \pm 7,3$ \\
\hline $\mathrm{T}_{3}$ & 10 & $66,66 \mathrm{~B}(10 / 15)$ & 10 & $31,5 \pm 3,0$ & 10 & $20,2 \pm 9,4$ \\
\hline
\end{tabular}

Valores seguidos por letras distintas na coluna diferem entre si pelo teste qui-quadrado $(\mathrm{P}<0,05)$.

Pierson et al. (2003) verificaram resultados similares ao do presente estudo, em relação à porcentagem de animais em estro $(66,6 \%)$, ao utilizarem $50 \mu \mathrm{g}$ de GnRH 24 horas após a remoção das esponjas em cabras fora da estação reprodutiva. Possivelmente, a administração de LH e GnRH neste estudo provocou efeito inibitório sobre a esteroidogênese ovariana. Os autores observaram que não houve diferença significativa no intervalo da retirada da esponja ao início do estro entre as cabras do tratamentocontrole $(45,7 \mathrm{~h})$ e as tratadas com GnRH $(39,3 \mathrm{~h})$. Os resultados deste estudo foram similares aos encontrados por esses autores, isto é, não houve diferença entre tratamentos.

Também não houve diferença $(\mathrm{P}>0,05)$ quanto à duração do estro entre os três tratamentos, cuja duração média foi de 19,7 horas. Fonseca (2002) observou $25 \mathrm{~h}$ de duração do estro com a utilização de esponjas impregnadas com $60 \mathrm{mg}$ de MAP por nove dias, associada a 200UI de eCG, e $22,5 \mu \mathrm{g}$ de prostaglandina sintética, aplicadas 24 horas antes da remoção do progestágeno.

A duração do estro observada neste estudo foi mais curta que a descrita por Fonseca (2002). Isso, possivelmente, pode ser atribuído ao momento da aplicação da eCG. Conforme Cameron e Batt, (1991), essa redução na duração do estro pode ter sido conseqüência da eCG, que promoveu a ocorrência de um pico préovulatório, induzindo a ovulação prematura.

No $\mathrm{T}_{1}$, todos os animais manifestaram estro. A maior proporção $(46,1 \%)$ foi observada entre 36 e 49 horas após a retirada das esponjas. A porcentagem de manifestação de estro para as cabras do $\mathrm{T}_{2}$ e $\mathrm{T}_{3}$ foi $53,3 \%$, entre 24 e 36 horas após a retirada das esponjas. Não foi observado estro em $26,6 \%$ e $33,3 \%$ das cabras do $\mathrm{T}_{2}$ e $\mathrm{T}_{3}$, respectivamente, até as 96 horas da retirada das esponjas. Baixas porcentagens de animais do $\mathrm{T}_{2}$ $(20,0 \%)$ e do $\mathrm{T}_{3}(13,3 \%)$ apresentaram estro antes das 24 horas, ou seja, antes da aplicação dos hormônios.

Os resultados deste estudo mostraram diferença com o uso do LH e do GnRH $(\mathrm{P}<0,01)$ em relação ao intervalo da retirada da esponja à ovulação (Tab. 2). Houve redução de, aproximadamente, nove horas com o uso do GnRH, mas não houve diferença de ambos com o $\mathrm{T}_{1}$. Cameron et al. (1988), ao aplicarem 50 $\mathrm{g}$ de GnRH, 20 horas após a remoção das esponjas, observaram $91,0 \%$ de ovulações ocorrendo entre 16 e 28 horas após. No presente estudo, não houve diferença $(\mathrm{P}>0,05)$ entre tratamentos no intervalo da aplicação de solução salina, ou de LH, ou de GnRH, à ovulação, mas ocorreu diferença $(\mathrm{P}<0,05)$ em relação ao intervalo do início do estro à ovulação entre as cabras do $\mathrm{T}_{2} \mathrm{e}$ $\mathrm{T}_{3}$ em comparação com as do $\mathrm{T}_{1}$.

$\mathrm{O}$ intervalo do início do estro à ovulação aumentou significativamente $(\mathrm{P}<0,05)$ com o uso do LH e GnRH em relação ao tratamentocontrole. Esse atraso no momento da ovulação pode ser conseqüência da formação de anticorpos anti-eCG, atribuído ao uso da eCG em pesquisas realizadas anteriormente no mesmo rebanho. De acordo com Roy et al. (1999a), os anti-eCG atrasam a onda pré-ovulatória de LH e o momento da ovulação. 


\section{Leite et al.}

Tabela 2. Intervalo da retirada da esponja à ovulação (IREO), intervalo da aplicação de solução salina $\left(\mathrm{T}_{1}\right)$, ou LH $\left(\mathrm{T}_{2}\right)$ ou GnRH $\left(\mathrm{T}_{3}\right), 24$ horas após a retirada da esponja, à ovulação (IAO) e intervalo do início do estro à ovulação (IEO) em cabras com estro induzido fora da estação reprodutiva com a utilização de MAP + eCG + cloprostenol (média \pm DP)

\begin{tabular}{|c|c|c|c|c|c|c|}
\hline \multirow{2}{*}{ Tratamento } & \multicolumn{2}{|c|}{ IREO } & \multicolumn{2}{|c|}{$\mathrm{IAO}$} & \multicolumn{2}{|c|}{ IEO } \\
\hline & $\mathrm{n}$ & horas & $\mathrm{n}$ & horas & $\mathrm{n}$ & horas \\
\hline$\overline{\mathrm{T}_{1}}$ & 13 & $46,6 \pm 9,3 \mathrm{ab}$ & 13 & $21,3 \pm 8,6$ & 13 & $11,7 \pm 14,1 \mathrm{~A}$ \\
\hline $\mathrm{T}_{2}$ & 15 & $52,1 \pm 5,1 \mathrm{a}$ & 15 & $26,8 \pm 4,1$ & 11 & $23,7 \pm 5,1 \mathrm{~B}$ \\
\hline $\mathrm{T}_{3}$ & 15 & $41,6 \pm 8,7 b$ & 15 & $22,3 \pm 13,3$ & 10 & $22,0 \pm 9,8 \mathrm{~B}$ \\
\hline
\end{tabular}

Médias na mesma coluna seguidas de letras distintas minúsculas $(\mathrm{P}<0,003)$ ou maiúsculas $(\mathrm{P}<0,05)$ diferem entre si pelo teste $\mathrm{SNK}$.

Pierson et al. (2001) observaram, também fora da estação reprodutiva, intervalo de 38,6 horas da retirada da esponja à onda de $\mathrm{LH}$, de 9,7 horas do início do estro à onda de $\mathrm{LH}$ e de 24 horas da onda de LH à ovulação. Levando-se em consideração que o intervalo médio da retirada das esponjas à ovulação foi de 46 horas e o intervalo da aplicação de solução salina, ou de LH ou de GnRH, à ovulação foi de 23 horas (Tab. 2), e que a duração do estro foi de 19 horas (Tab. 1), propõe-se que os hormônios sejam aplicados mais tardiamente para aproximar e potencializar o pico endógeno de $\mathrm{LH}$ e, assim, realizar a inseminação em um momento mais próximo à ovulação.

As cabras do $\mathrm{T}_{2}$ e $\mathrm{T}_{3}(73,3 \%)$ e do $\mathrm{T}_{1}(53,8 \%)$, em sua maioria, ovularam entre 40 e 55 horas após a retirada das esponjas. $\mathrm{O}$ diâmetro médio dos folículos ovulados foi de $8,2 \pm 1,0 \mathrm{~mm}$, e não houve diferença entre os tratamentos $(\mathrm{P}>0,05)$.

No $T_{1}$, das 13 cabras inseminadas, cinco $(38,4 \%)$ estavam gestantes quando se realizou o exame ultra-sonográfico, ao $25^{\circ}$ dia, e quatro $(30,7 \%)$ no exame feito no $40^{\circ}$ dia após a IA. No $\mathrm{T}_{2}$, das 15 cabras inseminadas, $11(73,3 \%)$ estavam gestantes aos 25 dias e cinco $(33,3 \%)$ aos 40 dias. No $\mathrm{T}_{3}$ foram encontradas os mesmos percentuais.

As taxas de gestação não diferiram entre os tratamentos $(\mathrm{P}>0,05)$, apesar de ser observada alta taxa de perda embrionária no $\mathrm{T}_{2}$ e $\mathrm{T}_{3}$ (Fig. 1). Nesses tratamentos, observou-se perda embrionária de $40,0 \%$ entre os dias 25 e 40 de gestação. Não houve perda fetal após os 40 dias de gestação, o que foi confirmado pelo diagnóstico realizado no $60^{\circ}$ dia após a IA.

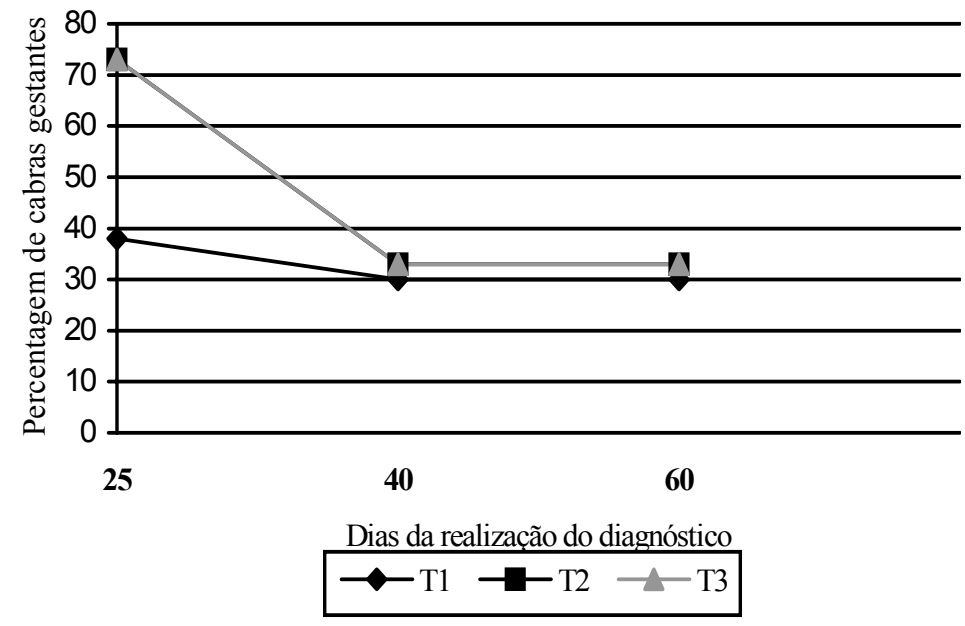

Figura 1. Diagnóstico de gestação em cabras com estro induzido fora da estação reprodutiva, com o uso de MAP + eCG + cloprostenol associado à aplicação de solução salina $\left(T_{1}\right)$, ou LH $\left(T_{2}\right)$ ou GnRH $\left(T_{3}\right)$, realizada 24 horas após a retirada da esponja. 
Neste estudo, a taxa de gestação não diferiu $(\mathrm{P}>0,05)$ entre os tratamentos, sendo obtida taxa de gestação média de 62,7\% aos 25 dias (Fig. 1). Zarkawi et al. (1999), ao trabalharem com cabras fora da estação reprodutiva, com a utilização de esponjas impregnadas com 60mg de MAP por 18 dias, e 200UI de eCG aplicadas na remoção da esponja, verificaram taxa de gestação de $65,8 \%$.

Deve-se também considerar os efeitos da IA em horário fixo após tratamento com eCG. De acordo com Roy et al. (1999a,b), existe variabilidade natural entre as fêmeas no momento da ovulação. Um atraso na onda préovulatória de LH em cabras tratadas anteriormente com eCG potencializa esse problema, pois cabras com alta concentração de anticorpo anti-eCG $(>2,5 \mu \mathrm{g} / \mathrm{ml})$ no momento da injeção exibiram baixa fertilidade após a IA em tempo fixo quando comparado a outras fêmeas $(41,3 \%$ vs $66,7 \%)$.

Freitas et al. (1997), ao estudarem fêmeas fora da estação reprodutiva, observaram taxa de gestação de $83,9 \%$ nos dias 21 e 22 , e $76,8 \%$, nos dias 45 e 46; esses autores também notaram perda embrionária entre os dias 21 e 45 após a IA. No presente estudo, perda embrionária de $40,0 \%$ nos tratamentos com LH e GnRH, ocorreu entre o $25^{\circ}$ e o $40^{\circ}$ dia após a IA (Fig. 1), podendo ter como causa a insuficiente secreção de progesterona nas etapas iniciais da prenhez, provocada por inadequado suporte luteotrófico do corpo lúteo (CL) e/ou sua luteólise prematura. Fonseca (2002), ao trabalhar com o mesmo rebanho e na mesma estação do ano, quando também se realizou este estudo, relatou perda embrionária antes dos primeiros 30 dias de gestação, não encontrando resposta para tal observação.

\section{CONCLUSÕES}

Conclui-se que a administração de LH ou GnRH após tratamento com esponjas impregnadas com MAP para induzir e sincronizar a ovulação em cabras fora da estação reprodutiva não apresenta resultados satisfatórios e não afeta a duração do estro.

\section{REFERÊNCIAS BIBLIOGRÁFICAS}

BARIL, G. Possibilidades atuais da transferência de embriões em caprinos. In: CONGRESSO BRASILEIRO DE REPRODUÇÃO ANIMAL, 11., 1995, Belo Horizonte. Anais... Belo Horizonte: CBRA, 1995.

BARIL, G.; LEBOEUF, B.; SAUMANDE, J. Synchronization of estrus in goats: the relationship between time of occurrence of estrus and fertility following artificial insemination. Theriogenology, v.40, p.621-628, 1993.

CAMERON, A.W.N.; BATT, P.A. PMSG may directly stimulate ovulation in female goats. Anim. Reprod. Sci., v.25, p.233-239, 1991.

CAMERON, A.W.N.; BATTYE, K.M.; TROUNSON, A.O. Time of ovulation in goats (Capra hircus) induced to superovulate with PMSG. J. Reprod. Fertil., v.83, p.747-752, 1988.

CHEMINEAU, P.; COGNIÉ, Y.; GUÉRIN, Y. Training manual on artificial insemination in sheep and goats. Rome: Italy, 1991. 222p.

FONSECA, J.F. Reprodução assistida em cabras. 2002. 105f. Tese (Doutorado em Zootecnia) Universidade Federal de Viçosa, Viçosa, MG

FREITAS, V.J.F.; BARIL, G.; SAUMANDE, J. Estrus synchronization in dairy goats: use of fluorogestone acetate vaginal sponges or norgestomet ear implants. Anim. Reprod. Sci., v.46, p.237-244, 1997.

FREITAS, V.J.F.; BARIL, G.; SAUMANDE, J. Induction and synchronization of estrus in goats: the relative efficiency of one versus two fluorogestone acetate-impregnated vaginal sponges. Theriogenology, v.46, p.1251-1256, 1996.

GONZÁLEZ, A.T. Controle da reprodução caprina. In: AGUIRRE, S.I.A. (Ed.). Producción de caprinos, 1986.

GREYLING, J.P.C.; van der NEST, M. Synchronization of oestrus in goats: dose effect of progestagen. Small Rum. Res., v.36, p.201207, 2000.

GREYLING, J.P.C.; van NIEKERK, C.H. Effect of pregnant mare serum gonadotrophin (PMSG) and route of administration after progestagen treatment on oestrus and LH secretion in the 
Boer goat. Small Rum. Res., v.3, p.511-516, 1990.

MENCHACA, A.; RUBIANES, E. Relation between progesterone concentrations during the early luteal phase and follicular dynamics in goats. Theriogenology, v.57, p.1411-1419, 2002.

MENEGATOS, J.; CHADIO, S.E.; KARATZAS, G. et al. Progesterone levels throughout progestagen treatment influence the establishment of pregnancy in the goat. Theriogenology, v.43, p.1365-1370, 1995.

MOTLOMELO, K.C.; GREYLING, J.P.C.; SCHWALBACH, L.M.J. Synchronization of oestrus in goats: the use of different progestagen treatments. Small Rum. Res., v.45, p.45-49, 2002.

PIERSON, J.T.; BALDASSARRE, H.; KEEFER, C.L. et al. Influence of GnRH administration on timing of the LH surge and ovulation in dwarf goats. Theriogenology, v.63, p.1-10, 2003.

PIERSON, J.T.; BALDASSARRE, H.; KEEFER, C.L. et al. Seasonal variation in preovulatory events associated with synchronization of estrus in dwarf goats. Theriogenology, v.56, p.759-769, 2001.
ROY, F.; COMBES, B.; VAIMAN, D. et al. Humoral immune response to equine chorionic gonadotropin in ewes: association with major histocompatibility complex and interference with subsequent fertility. Biol. Reprod., v.61, p.209218, $1999 b$.

ROY, F.; MAUREL, M-C.; COMBES, B. et al. The negative effect of repeated equine chorionic gonadotropin treatment on subsequent fertility in alpine goats is due to a humoral immune response involving the major histocompatibility complex. Biol. Reprod., v.60, p.805-813, 1999a.

RUBIANES, E.; CASTRO, T.; CARBAJAL, B. Effect of high progesterone levels during the growing phase of the dominant follicle of wave 1 in ultrasonically monitored ewes. Can. J. Anim. Sci., v.76, p.473-475, 1996.

SHELTON, M. Reproduction and breeding of goats. J. Dairy Sci., v.61, p.994-1010, 1978.

SISTEMA de análises estatísticas e genéticas SAEG. Viçosa, MG: UFV, 1999 (versão 8.x).

ZARKAWI, M.; AL-MERESTANI, M.R.; WARDEH, M.F. Induction of synchronized oestrous in indigenous Damascus goats outside the breeding season. Small Rum. Res., v.33, p.193-197, 1999. 